Muthinja, M. J. et al. (2017) Microstructured blood vessel surrogates reveal structural tropism of motile malaria parasites. Advanced Healthcare Materials, 6(6), 1601178. (doi:10.1002/adhm.201601178)

There may be differences between this version and the published version. You are advised to consult the publisher's version if you wish to cite from it.

This is the peer-reviewed version of the following article: Muthinja, M. J. et al. (2017) Microstructured blood vessel surrogates reveal structural tropism of motile malaria parasites. Advanced Healthcare Materials, 6(6), 1601178, which has been published in final form at 10.1002/adhm.201601178. This article may be used for noncommercial purposes in accordance with Wiley Terms and Conditions for Self-Archiving.

http://eprints.gla.ac.uk/138084/

Deposited on: 09 March 2017

Enlighten - Research publications by members of the University of Glasgow http://eprints.gla.ac.uk 
DOI: 10.1002/ adhm.201601178R1

Article type: Full Paper

\section{Micro-structured Blood Vessel Surrogates Reveal Structural Tropism of Motile Malaria Parasites}

Julianne M. Muthinja, Johanna Ripp, Janina K. Hellmann, Tamas Haraszti, Noa Dahan, Leandro Lemgruber, Anna Battista, Lucas Schütz, Oliver T. Fackler, Ulrich S. Schwarz, Joachim P. Spatz and Friedrich Frischknecht

J. M. M. Author 1, J. R. Author 2, Dr. J. K. H. Author 3, Dr. T. H. Author 4, Dr. N. D. Author

5, Dr. L. L. Author 6, Dr. A. B. Author 7, L. S. Author 8, Prof. O. T. F. Author 9, Prof. U. S.

S. Author 10, Prof. J. P. S. Author 11, Prof. F. F. Author 12

J. M. M. Author 1, J. R. Author 2, Dr. J. K. H. Author 3, Dr. N. D. Author 5, Dr. L. L. Author 6, L. S. Author, Prof. F. F. Author 12

Integrative Parasitology, Center for Infectious Diseases, Heidelberg University Hospital, Im Neuenheimer Feld 324, 69120 Heidelberg, Germany

Phone: +49-6221-566537 Fax: +49-6221-564643

Email: freddy.frischknecht@med.uni-heidelberg.de

Dr. T. H. Author 4, Prof. J. P. S. Author 11

Department of Biointerphase Science \& Technology, Max Planck Institute for Medical Research and Laboratory of Biophysical Chemistry, Heidelberg University; Jahnstrasse 29, 69120 Heidelberg, Germany 
Prof. O. T. F. Author 9

Integrative Virology, Center for Infectious Diseases, Heidelberg University Hospital, Im Neuenheimer Feld 324, 69120 Heidelberg, Germany

Dr. A. B. Author 7, Prof. U. S. S. Author 10

Theoretical Physics and BioQuant, Heidelberg University, Im Neuenheimer Feld 267, 69120

Heidelberg, Germany

Keywords: PDMS pillar arrays, gliding motility, sporozoites, apicomplexa, blood vessels 


\begin{abstract}
Plasmodium sporozoites, the highly motile forms of the malaria parasite, are transmitted naturally by mosquitoes and traverse the skin to find, associate with, and enter blood capillaries. Research aimed at understanding how sporozoites select blood vessels was hampered by the lack of a suitable experimental system. Arrays of uniform cylindrical pillars can be used to study small cells moving in controlled environments. Here we developed an array system displaying a variety of pillars with different diameters and shapes in order to investigate how Plasmodium sporozoites associate to the pillars as blood vessel surrogates. Investigating the association of sporozoites to pillars in arrays displaying pillars of different diameters revealed that the crescent-shaped parasites prefer to associate with and migrate around pillars with a similar curvature. This suggests that after transmission by a mosquito malaria parasites might use a structural tropism to recognize blood capillaries in the dermis in order to gain access to the blood stream.
\end{abstract}




\section{Introduction}

The generation of artificial 2D substrates and 3D environments has improved our toolkit for the study of cell adhesion and migration. ${ }^{[1-3]}$ Available $2 \mathrm{D}$ substrates include adhesive micropatterns or nanoscopic ligand binding sites or topological features, like a 'beds of needles'. ${ }^{[4,5]}$ The latter, also called pillar arrays, are generated by fabricating cylindrical micropillars from the crosslinked polymer gel polydimethylsiloxane (PDMS) in defined geometries. ${ }^{[1,4,6,7]}$ By coating the pillar tops with adhesive ligands and tuning pillar stiffness to cell forces, such arrays are commonly used measure cellular traction forces. ${ }^{[8,9]}$ We have previously adapted pillar arrays for the investigation of small motile cells that can be placed between the pillars. ${ }^{[10]}$ In these arrays, the uniform pillars do not serve as substrates for cell adhesion but as obstacles for the migrating cells, which can be different types of eukaryotic or bacterial cells. ${ }^{[10-15]}$ These studies showed that the distance between the pillars affects the migration patterns of cells.

As a first model cell we used the form of a rodent malaria parasite that is transmitted from the mosquito vector to the mammalian host, i.e. the Plasmodium sporozoite. Sporozoites are deposited during the mosquito bite in the dermis of the host where they migrate and enter into blood capillaries. ${ }^{[16,17]}$ Sporozoite migration is essential for infectivity of the parasite, which ultimately needs to enter the liver to further differentiate into blood cell infecting parasite forms that cause the disease ${ }^{[18]}$ One open question concerning the transmission of the malaria parasite is how the parasite finds and associates with blood capillaries. We have suggested that sporozoite migration in the skin is independent of chemotaxis and relies mainly on the structure of the environment. ${ }^{[10]}$ Furthermore, Plasmodium sporozoites are curved cells with a curvature that is similar to the curvature of the blood capillaries they enter. ${ }^{[19,20]}$ In vivo experiments analyzing the tracks of many parasites further showed that sporozoites migrate with slightly reduced speed around capillaries compared to their migration in the surrounding tissue. $^{[21]}$ Around blood capillaries they also adapt a more uniform curvature that appears 
different from the surrounding tissue, where they often change their shape. ${ }^{[21]}$ These observations led us to the hypothesis that sporozoites develop their curvature to select blood vessels of a matching curvature. To test this hypothesis, we sought to generate PDMS pillars of different diameters as simple blood vessel surrogates in order to probe if sporozoites would specifically associate with pillars of a defined curvature even in the absence of molecular cues. To this end we generated a set of new pillar arrays each displaying pillars of two different diameters. In addition we designed a set of pillars that deviate from the circular form. In this second set of arrays, circular pillars were mixed with squared and pentagon-shaped pillars to test if sporozoite adhesion to these pillars was depending on their shape.

\section{Results}

We first generated a set of PDMS pillar arrays with pillar diameters between 6 and $14 \mu \mathrm{m}$. In each array pillars of two different diameters were placed next to each other in an orthogonal fashion (Figure 1). Since the different diameters of pillars caused an uneven distribution of pillar-to-pillar distances in different directions of each pillar, we generated two types of patterns for most pairs of pillars. One pattern was produced such that a pillar with the larger diameter (thicker pillar) was surrounded by 6 pillars with a smaller diameter (thinner pillars) and thus resulting in 3 times more of the thinner pillars within the overall pillar pattern. The other pattern was produced such that one thinner pillar was surrounded by 6 thicker pillars (Figure 1B). This allowed us to correct for potential bias towards association around overrepresented pillars and potential problems in the migration behavior of the cells placed between the pillars (see below). Taking this precaution was necessary, as we had previously shown that the migration pattern of sporozoites could vary as a function of pillar-to-pillar distance. ${ }^{[10]}$ In arrays where a larger number of thicker pillars were present the available space for movement between pillars was smaller than in arrays featuring a majority of thinner pillars (Figure 1B). For example, in an array featuring pillars with 8 and $10 \mu \mathrm{m}$ diameters, the 
available area between pillars was $40 \%$ of the total array surface in arrays with a majority of $10 \mu \mathrm{m}$ pillars. However, the area between pillars was $45 \%$ of the total array surface in the array with a majority of $8 \mu \mathrm{m}$ pillars. Analysis of the pillar arrays by scanning electron microscopy revealed that pillars were of regular height and circumference (Figure 1A,C). Sporozoites could be added to the pillar arrays in medium containing $3 \%$ bovine serum albumin (BSA) after wetting the pillar as described previously. ${ }^{[10]}$ We assume that BSA in the medium sticks to the PDMS or activates sporozoites, which can move on a wide variety of substrates. ${ }^{[22-24]}$ Sporozoites could associate with pillars in different ways: they could be found on the top (Figure 1C, top panel), at the side of (Figure 1C, middle panel) and between pillars (Figure 1C, bottom panel).

We next observed motile sporozoites expressing the green fluorescent protein (GFP) in their cytoplasm by wide-field or spinning disc confocal microscopy. Sporozoites were found to migrate in different fashions between, around and on top of the pillars (Figure 2, Figure S1 and Movie S1 and S2). Sporozoites could migrate between pillars in a meandering and linear fashion as described for uniform pillar arrays (Figure S1A). ${ }^{[10]}$ Sporozoites could also move around pillars (Figure 2A). Most sporozoites moving around pillars with a diameter of $>8$ micrometers were tightly attached to the pillars. In contrast only half of 136 observed sporozoites moving around pillars of $6 \mu \mathrm{m}$ diameter associated tightly with these pillars while the other half was moving at a visible distance from the pillar in a circular fashion (Figures 2A,B, Figure S1B). Sporozoites failed to closely associate with pillars of $4 \mu \mathrm{m}$ diameter. Sporozoites moving in pillar arrays with pillars of 4 or $6 \mu \mathrm{m}$ diameter also moved around several pillars on larger circular tracks (Figure 2C). Sporozoites could move around one pillar, dissociate from it and associate to a different pillar (Figure 2D and Figure S1A). Sporozoites could also move on top of large pillars (Figure 2E, Figure S1C,D). On top of the pillars sporozoites could also float in the medium (Figure 2F). This could lead to the highlighting of the pillar circumference if the fluorescent sporozoites were momentarily on 
top of the pillars. This lensing effect needed to be accounted for during analysis with maximum fluorescent projections as it mimicked the pattern of sporozoite circling around the pillars (Figure S1E and Movie S3).

We next quantified how many sporozoites moved around thinner and thicker pillars. To this end two students counted a set of experiments independently from each other. We observed a different distribution of sporozoites preferentially associating with either thinner or thicker pillars in some of the arrays (Figure 3). This revealed that $P$. berghei sporozoites preferred to move around the thicker pillar in arrays presenting pillars with 6 and $10 \mu \mathrm{m}$ diameter. In contrast, sporozoites preferred to move around the thinner pillars when pillars of 10 and 14 $\mu \mathrm{m}$ diameter were present (Figure 3C). Curiously, in arrays of 8 and $12 \mu \mathrm{m}$ diameter we observed a preference for $8 \mu \mathrm{m}$ pillars only in arrays where the $8 \mu \mathrm{m}$ pillar was surrounded by $12 \mu \mathrm{m}$ pillars, but no difference in the reciprocal array (Figure 3C). No difference was observed in arrays with pillars of 8 and $10 \mu \mathrm{m}$ diameters, while sporozoites appear to slightly prefer moving around $10 \mu \mathrm{m}$ pillars in arrays of pillars with 10 and $12 \mu \mathrm{m}$ diameter (Figure 3D). A preference for $12 \mu \mathrm{m}$ pillars was observed in arrays containing pillars of 12 and $14 \mu \mathrm{m}$ diameter (Figure 3D). Intriguingly, sporozoites of the related rodent malaria parasite Plasmodium yoelii preferred to move around pillars of $12 \mu \mathrm{m}$ diameter in arrays containing pillars of 12 and $8 \mu \mathrm{m}$ as well as 12 and $10 \mu \mathrm{m}$ diameters (Figure S2). We lastly compared sporozoite association in 6-10 $\mu \mathrm{m}$ pillar arrays with different pillar-to-pillar distances (Figure 3E, F). This showed a similar distribution of sporozoites independent from the pillar-to-pillar distance, which varied from 6 to $10 \mu \mathrm{m}$ (Figure 3E, F). Additionally, we observed that the proportion of circular gliding sporozoites in 6 and 10 um arrays decreased over time and was not different from sporozoites gliding on a flat PDMS support (Figure S3). Taking these data together, we conclude that $P$. berghei sporozoites move preferentially around pillars with a diameter between 8 and $12 \mu \mathrm{m}$, which is similar to their own curvature and the diameter of 
blood capillaries in the skin. ${ }^{[25]}$ Curiously $P$. yoelii sporozoites, which are slightly larger than P. berghei sporozoites but show a similar curvature, prefer slightly larger pillars.

We next aimed to determine the natural distribution of curvatures in a P. berghei sporozoite population and test if they correlated with other parameters such as sporozoite length or speed. Two different methods of measurements of length and curvature yielded slightly different but consistent sets of data. Manual (Figure S4) and automated analysis (Figure 4) showed an average length of $10.3 \pm 1.4 \mu \mathrm{m}$ (range: $3.3-17.2 \mu \mathrm{m}$ ) versus $11.6 \mu \mathrm{m}$ (range: 8 to $14.5 \mu \mathrm{m}$ ), respectively. Sporozoites showed a curvature of $0.15 \pm 0.05 \mu \mathrm{m}^{-1}$ (range: $0.003-0.41 \mu \mathrm{m}^{-1}$ ) versus $0.22 \mu \mathrm{m}^{-1}$ (range: 0.13 to $0.35 \mu \mathrm{m}^{-1}$ ), corresponding to a diameter of the trajectory resulting from their migration of $13 \mu \mathrm{m}$ and $9 \mu \mathrm{m}$, respectively. Interestingly, P. yoelii sporozoites were observed to be longer with a mean of $12.5 \mu \mathrm{m}$ (range: 8.5 to $16.0 \mu \mathrm{m}$ ) but showed the same curvature (mean 0.23 ; range: 0.13 to $0.35 \mu \mathrm{m}^{-1}$ ), compared to $P$. berghei when using the automated method (Figure 4). Individual sporozoite length did not correlate with curvature if the entire population was investigated. However, when we investigated the 2 to 7 percent of parasites that were particularly long, we found their curvature to be significantly smaller. Particularly short sporozoites showed higher curvatures (Figure 4C, Figure S4). Thus, in the extremes, sporozoite length was found to inversely correlate with curvature.

This raised the question if sporozoites circling around thin pillars are shorter than those circling around thick pillars. Indeed, when investigating sporozoites moving around $6 \mu \mathrm{m}$ pillars and those moving around $14 \mu \mathrm{m}$ pillars, we found the former to be on average $2 \mu \mathrm{m}$ shorter than the latter (Figure 5A). We next determined the speed with which sporozoites migrated around the various pillars. We found that sporozoites moving around pillars of $6 \mu \mathrm{m}$ diameter were slower than those moving on flat substrates, while there was no difference between the latter and sporozoites moving around pillars of $14 \mu \mathrm{m}$ diameter. Curiously, the speed appeared only slightly higher for sporozoites going around pillars with $14 \mu \mathrm{m}$ diameter 
compared with pillars of $6 \mu \mathrm{m}$ diameter but this difference was not statistically significant with a P-value of 0.12 (Figure 5B). This suggests that only subtle modulation in speed might occur when sporozoites associate with certain pillars. More importantly these data suggest that association to a circular object per se is not enough to slow down sporozoites and does not change the persistence of sporozoite motility. In vivo one can observe that sporozoites slow down prior to blood vessel entry suggesting that a specific molecular interaction is important for this step. ${ }^{[26]}$

To further investigate the flexibility of sporozoites to associate with pillars we generated a set of pillars with very large pillar diameters (Figure 6). This showed fewer sporozoites migrating around pillars of $18-20 \mu \mathrm{m}$ diameters compared with pillars of smaller diameters (Figure 6B). Instead, sporozoites would associate with the side or top of pillars and use these surfaces as substrates to perform gliding motility (Figures 6C, 2E and Movie S2). This suggests that sporozoites can tolerate only a certain degree of deformation and prefer to migrate with a given curvature determined by their internal organization. This is interesting as in vivo sporozoites only invade the thin capillaries and not the larger blood vessels, thus hinting that sporozoite curvature might allow parasites to de-select from associating to these bigger vessels.

To test if the presence of pillars and thus a more confined environment might induce motility, we next investigated sporozoites that were not capable to migrate. To this end we put midgutderived sporozoites into a set of pillar arrays and also investigated sporozoites lacking the actin filament binding protein coronin, which show defects in $2 \mathrm{D}$ but not in $3 \mathrm{D}$ motility. ${ }^{[27]}$ However, neither of these sporozoite populations showed an increase of their migration capacity in the pillar arrays (Figure 7).

Lastly, we sought to probe whether the shape of the object would influence sporozoite motility around pillars. We argued that a squared or pentagon-shaped pillar would allow the parasite to attach less efficiently to the pillar compared to circular pillars. To this end we 
generated a set of pillars where circular and differently shaped pillars were inter-spaced (Figure 8A). In a first attempt we designed the different shapes such that the diameter of the pillars was $10 \mu \mathrm{m}$. We set the outer diameter of the differently shaped pillars to $10 \mu \mathrm{m}$. However, this turned out to yield pillars that were too small for sporozoites to attach. During moulding of the PDMS substrate, the structures would shrink and sporozoites would not attach to them but simply glide in circles around them, similarly to what was observed with thin pillars (Figure 2B). We thus designed a further set of pillar substrates where the outer diameter of the pillars was $11 \mu \mathrm{m}$. Sporozoites migrated in the resulting pillar arrays and associated either with circular or differently shaped pillars. While there was no discernible difference in average sporozoite speed, we observed that sporozoites moving around the differently shaped pillars would move in a slightly different way. This was most pronounced around the square shaped pillars, where about half of the sporozoites changed their shape by stretching, resulting in a somewhat squared trajectory (Figures 8B,C). Taken all presented data together, these observations suggest that sporozoites can only slightly adapt their curvature to the pillars and prefer those with a matching curvature. In this way, sporozoites could select for the small capillaries in the skin that they invade after transmission by a mosquito bite. 


\section{Discussion}

Here, we present a set of new PDMS micropillar arrays and probed their utility to answer a biological question important for our understanding of malaria parasite transmission. In general our results show that micro-structured mixed pillar arrays can be used for determining potentially important parameters within a given cell population. The number of individual parasites that could be probed was at least two orders of magnitude higher than what could have been observed in a similar time frame for in vivo experiments, where the tissue environment is never the same. The highly similar experimental in vitro setting in our pillar arrays thus allowed the investigation of subtle differences that would likely be impossible to observe in vivo. Hence our approach will be interesting for the quantitative study of many other bacterial and eukaryotic cells that migrate in complex natural environments. ${ }^{[28]}$ Based on our observations we suggest that Plasmodium sporozoites have adapted a crescent shape partly in order to associate with circular objects, potentially the blood capillaries that they need to penetrate minutes after being transmitted by the mosquito. Interestingly, our data suggest that no other molecular cue is important for this initial association. Yet, the comparison of the speeds of sporozoites moving around pillars of different sizes hints that ligand-receptor interactions must play a role to slow sporozoites in vivo. Importantly, both human infecting malaria parasites $P$. falciparum and $P$. vivax show sporozoites with similar curvature to those of our model rodent parasites. ${ }^{[29]}$ Blood capillaries of humans and mice measure about $10 \mu \mathrm{m}$ in diameter and indeed sporozoites were found to circle around capillaries prior to entering them when imaged in vivo while they are rarely seen migrating around the much larger blood vessels that can also be observed in the dermis. ${ }^{[26,30]}$ However these in vivo data are hard to quantify and different mechanisms such as receptor ligand pairing might also contribute to capillary selection. Intriguingly we also found fewer 
sporozoites circling around larger pillars suggesting that their curvature cannot be adapted to migrate around these or indeed around large blood vessels in vivo.

We have recently shown that the curvature of the sporozoite is likely due to the establishment of a cytoskeletal structure, the so-called sub-pellicular network. ${ }^{[19]}$ This structure can be visualized with cryo-electron tomography in mature sporozoites isolated from the mosquito salivary glands, while it is absent from immature sporozoites isolated from parasitic cysts of the mosquito gut, where sporozoites form. ${ }^{[19,31]}$ Only mature sporozoites are able to attach efficiently to a flat substrate and perform robust motility. ${ }^{[32,33]}$ Our current dataset suggests that sporozoites have a given curvature that is normally distributed over a certain range and would not fully adapt their curvature to objects they associate with. This is somewhat surprising as sporozoites are flexible cells that can readily be deflected or bend by rigid obstacles in vitro and in vivo. ${ }^{[10,34,35]}$ On the other hand, it might well be that the intracellular cytoskeleton allows only a temporal bending of the sporozoite during a rapid encounter with an obstacle or when forced to go through a narrow channel, as is the case in the salivary gland. ${ }^{[34]}$ The intracellular cytoskeleton might not allow for long-term adjustment of the shape and force the parasite back to its given shape as soon as the obstacle is bypassed or the channel widens. Indeed such relaxation can be observed in salivary canals, when straightened sporozoites move down the narrow ducts and arrive at the wider salivary canals: sporozoites immediately return to their crescent shape. ${ }^{[34]}$ Thus, the parasite would adopt only one given shape as its energetic 'ground state' as determined by the intracellular cytoskeleton. This is somewhat similar to red blood cells, where a membrane-associated cytoskeleton determines an energetically favored biconcave shape but allows for extreme flexibility of the cell in response to external constraints. Curiously, the longer $P$. yoelii sporozoites showed the same overall curvature as $P$. berghei sporozoites but preferred to circle around slightly thicker pillars. This might indicate that the intracellular cytoskeleton is weaker in $P$. yoelii 
sporozoites allowing for more flexibility. However in absence of any physical measurements of parasite elasticity, this remains speculative.

To probe if sporozoites can actively adapt to a given shape in the absence of the subpellicular network, we also imaged immature sporozoites in our new mixed-pillars arrays. To do so we isolated immature sporozoites from the oocysts residing at the gut wall, which can only weakly adhere and basically do not move on substrates and are mostly thinner and longer then salivary gland sporozoites. ${ }^{[19,32]}$ When placed between pillars, the midgut sporozoites did not migrate (Figure 7). Only few showed a type of motility previously described as patch-gliding, where parasites are attached to the substrate with a single adhesion site and move over this adhesion site back and forth. ${ }^{[2]}$ No change in the capacity of midgut sporozoites to glide was observed in a variety of pillar arrays with different pillar diameters and pillar-to-pillar distances. This suggests that sporozoites do not actively adopt their shape but that the shape is determined during developmental maturation as is their capacity to attach to substrates and perform gliding. ${ }^{[32,33]}$ It also shows that our new mixed-pillar arrays can in principle be used to investigate different developmental stages of a cell, in our case the rodent relatives of the medically relevant human malaria parasite. Similarly, salivary gland-derived sporozoites lacking the actin filament binding protein coronin that show a defect in motility in $2 \mathrm{D}$ could not be induced to migrate robustly in pillar arrays with a pillar-to-pillar distance of 3-4 $\mu \mathrm{m}$ (Figure 7). These coronin(-) sporozoites move in the dermis similar to wild type sporozoites. ${ }^{[27]}$ This suggests that the confinement of the dermal tissue is higher than that conferred by the pillar arrays.

In vivo imaging of sporozoites in living mice found that sporozoites move slower on blood capillaries than in the nearby dermis with sporozoites moving across the dermis at $1.38 \mu \mathrm{m} / \mathrm{s}$ and those associated with blood vessels moving at $1.06 \mu \mathrm{m} / \mathrm{s} .{ }^{[21]}$ Intriguingly, we also found that sporozoites decrease their speed when circling around thinner pillars compared to larger pillars or when gliding in the absence of pillars (Figure 5B). This could hint towards the 
existence of a mechanism that slows sporozoites down when they attach to an object with matching curvature, possibly by allowing the formation of adhesion sites on the surface of the object (a pillar in vitro and a blood vessel in vivo). To limit the capacity to form adhesions we generated pillars with a non-circular shape (Figure 8). Sporozoites could readily associate with these pillars and move around them, but showed slightly deformed trajectories. This shows their flexibility and could possibly be used to analyse mutant parasite in the future to investigate differences in cellular elasticity. However, it also limited the comparative analysis of the speeds with sporozoites moving around circular pillars. We thus cannot conclude whether the shape of the pillars modulates sporozoite speed as much as can be observed in vivo, where at least some sporozoites completely arrest prior to entering the blood vessels. ${ }^{[26]}$ While we here probed the association of sporozoites to 'naked' structures, the blood capillaries in vivo are naturally surrounded by a number of components of the basal membrane i.e. different proteins and glycans. These could interact with the sporozoite and slow it down even further by either increasing adhesion efficiency or by signaling. ${ }^{[22,27,36,37]}$ This could be tested in pillar arrays or on flat substrates by linking the proteins covalently to the surface and comparing the speed of sporozoites gliding on these variable surfaces.

\section{Conclusions}

In conclusion, using a series of mixed-pillar arrays we were able to probe the capacities of Plasmodium sporozoites to associate with the outside of defined curved structures mimicking the shape of blood vessels. This shows a proof-of-principle for the utility of mixed-pillar arrays for the investigation of motile cells with their environments. The arrays should thus be interesting for different applications where cell function or shape is perturbed by drugs or protein knock-down (e.g. by RNAi) or when cells with depleted genes are investigated. The pillar arrays can likely also be employed in true $3 \mathrm{D}$ if the space between them is filled with a synthetic or natural matrix such as polyacrylamide, collagen or matrigel. Furthermore the 
mixed pillar arrays will likely be useful tools to dissect differences in adhesion behaviour in large mammalian cells that are grown on top of the pillars. ${ }^{[38]}$ Using the mixed pillar array we were able to determine the preferential pillar size for Plasmodium sporozoites association. We found that sporozoites tend to associate better with pillars of the size of blood capillaries in the dermis. This observation suggests the existence of a structural tropism of Plasmodium parasites that favours association to blood capillaries during transmission. This hypothesis could be tested in the future by a mix of in vivo experiments and our arrays, when genetically modified Plasmodium sporozoites are available that show a changed curvature while still migrating at the same speed.

\section{Materials and methods}

\subsection{Pillar array fabrication}

We designed a master mask using a home-written Python script and visualized it with the converter software of the DWL-66 mask writer (Heidelberg Instruments). The individual pillars were arranged in hexagonal patterns to assure a minimal deviating distance between pillars in any direction (Figure 1). Each pillar of one kind of diameter was surrounded by a hexagon of pillars of the other diameter, creating a heterogeneous array. The master masks were produced by ML\&C Jena and J.D. Photo-Tools UK. The PDMS micropillar substrates were then made by photolithographic and replicate molding techniques essentially as described in. ${ }^{[7,39]}$ To allow wetting of the hydrophobic pillar arrays they were first incubated with an alkaline solution containing 10\% Extran (Merck) in water for 20 minutes and gently shaken. Following, the structures were washed three times for 10 minutes in distilled water. A silicon flexiPERM chamber (GreinerBioOne) was used for imaging parasites within pillar arrays. The chamber surrounds the pillar arrays and maintains a stable environment without flow. ${ }^{[10]}$ Sporozoites in RPMI containing 3\% bovine serum albumin were added to the flexiPERM chamber surrounding a single pillar field. 


\subsection{Preparation of Plasmodium sporozoites}

Plasmodium berghei (strain NK65) wild type or Plasmodium berghei (strain ANKA) coronin(-) sporozoites constitutively expressing cytoplasmic green fluorescent protein (GFP) or mCherry under the control of the sporozoite-specific circumsporozoite protein promoter and Plasmodium yoelii sporozoites constitutively expressing cytoplasmic GFP under the control of the EF1a promoter were produced in Anopheles stephensi mosquitoes as described. ${ }^{[10,40]}$ For all experiments midgut sporozoites were used from day 15 to day 18 after mosquito infection and salivary gland derived sporozoites were used between day 17 and day 23 post-infection. Salivary glands and midguts were dissected in RPMI culture medium supplemented with $3 \%$ bovine serum albumin. Both organs were smashed with a plastic pestle in an Eppendorf reaction tube to release the sporozoites, which were further purified from debris by brief centrifugation ( $500 \mathrm{rpm}$ on an Eppendorf $5417 \mathrm{R}$ ) at $4^{\circ} \mathrm{C}$ for $5 \mathrm{~min}$. The use of mice for mosquito infection was approved by the German authorities (Regierungspräsidium Karlsruhe, Germany), $\S 8$ Abs. 1 Tierschutzgesetz (TierSchG) and all animal experiments were performed according to FELASA category B and GV-SOLAS standard guidelines.

\subsection{Light Microscopy}

Imaging was performed on a wide-field inverted Axiovert 200M Zeiss microscope using a GFP filter set or with a spinning disc confocal unit (PerkinElmer Inc.) on an Eclipse Ti inverted microscope (Nikon) at room temperature. Images on the Axiovert were collected with a Zeiss Axiocam HRM every 3 seconds with an exposure of $150 \mathrm{~ms}$ using Axiovision 4.6 software and a 10X Apoplan objective (N.A. 0.5). A time lapse of at least 2 minutes for each region of interest [ROI] was recorded. At least 20 sporozoites for each ROI were imaged per tested pillar diameter with usually over 200 sporozoites tested per array. Images on the spinning disc confocal were recorded on a Hamamatsu Orca Flash 4 (resolution) camera using the $20 \mathrm{X}$ objective (N.A. 0.75). All image series were imported to ImageJ for analysis. The movies were analysed with regard to pillar association by counting the numbers of parasites in 
a given movie circling around a pillar. To correct for analysis errors, datasets were analysed independently by two of the authors. Speed and curvature were analyzed by applying the ImageJ plugins Manual Tracking, mTrack2 or ToAST. ${ }^{[10,41]}$ Curvature was in addition manually determined by measuring the diameter of the circle described by the moving sporozoite and by tracing the front, center and rear position. The latter method gave lower curvature readings.

\subsection{Scanning Electron Microscopy}

Sporozoites were allowed to glide on different pillar arrays for 5-10 min at room temperature. The material was then fixed with $2.5 \%$ glutaraldehyde and $4 \%$ paraformaldehyde in $0.1 \mathrm{M}$ cacodylate buffer, $\mathrm{pH} 7.2$ for 30 minutes. Afterwards it was post-fixed at room temperature for $1 \mathrm{~h}$ in a solution containing $1 \% \mathrm{OsO}_{4}$ in $0.1 \mathrm{M}$ cacodylate buffer, $\mathrm{pH}$ 7.2. The material was washed, dehydrated in an ethanol series, critical point-dried in $\mathrm{CO}_{2}$ and mounted on specimen stubs. Stubs were sputtered with a thin layer of gold/palladium and observed in a Zeiss LEO 1530 scanning electron microscope.

\subsection{Statistics}

GraphPad Prism was used for graphing and statistical analysis. Mean and standard deviations were plotted for each graph if not stated otherwise and Students t-test was performed. Multiple means were compared using one-way analysis of variance (ANOVA), $p<0.05$ was considered statistically significant. Proportions were arranged in contingency tables and analyzed using Fischers exact test or a Chi-square test.

\section{Supporting Information}

Supporting Information is available from the Wiley Online Library or from the author.

\section{Acknowledgements}

We thank Miriam Reinig for mosquito infections, Leanne Strauss, Christian Sommerauer and several undergraduate students for help with analysis, Timo Maier and Ada Cavalcanti-Adam 
for help and discussions, Mirko Singer, Rogerio Amino and Photini Sinnis for discussions and reading the manuscript. We thank Christophe Zimmer for first pointing out the similarity between capillary and sporozoite curvature. The work was funded by grants from the German Federal Ministry of Education and Research (BMBF, Biofuture and NGFN), the Chica and Heinz Schaller Foundation, the Frontier Program of the University of Heidelberg, the German Research Foundation (DFG) through SFB 1129 and the European Research Council (StG 281719) and the Max Planck Society. We gratefully acknowledge support from the Medical School and the Electron Microscopy Unit at the University of Heidelberg for access to their microscopes. Author 1 received a $\mathrm{PhD}$ fellowship from the German Academic Exchange Service (DAAD). Authors 1, 2 and 8 are and author 3 was a member of the Hartmut Hoffmann-Berling International Graduate School at Heidelberg University. Author 5 held a Minerva fellowship from the Max Planck Society and a postdoctoral fellowship from the Alexander von Humboldt foundation. Author 6 was a recipient of a postdoctoral fellowship from the Cluster of Excellence CellNetworks at the Heidelberg University. Author 7 was a member and obtained a fellowship through the Heidelberg Graduate School of Mathematical and Computational Methods for the Sciences. Author 9, Author 10, Author 11 and Author 12 are members of the CellNetworks cluster of excellence at Heidelberg University and of SFB 1129. Author 12 was a member of the EU FP7 Network of Excellence EVIMalaR. Authors $2,3,4,5$ and 6 contributed equally to this work.

Received: ((will be filled in by the editorial staff))

Revised: ((will be filled in by the editorial staff)) Published online: ((will be filled in by the editorial staff)) 


\section{References}

[1] J. L. Tan, J. Tien, D. M. Pirone, D. S. Gray, K. Bhadriraju, C. S. Chen, Proc Natl Acad Sci $U S A$ 2003, 100, 1484.

[2] J. Fu, Y. K. Wang, M. T. Yang, R. A. Desai, X. Yu, Z. Liu, C. S. Chen, Nat. Methods 2010, $7,733$.

[3] Y. K. Wang, C. S. Chen, J. Cell. Mol. Med. 2013, 17, 823.

[4] J. le Digabel, M. Ghibaudo, L. Trichet, A. Richert, B. Ladoux, Med Biol Eng Comput 2010, $48,965$.

[5] M. G. Whitesides, E. Ostuni, S. Takayama, X. Jiang, E. D. Ingber, Annu. Rev. Biomed. Eng. 2001, 3, 335.

[6] N. Q. Balaban, U. S. Schwarz, D. Riveline, P. Goichberg, G. Tzur, I. Sabanay, D. Mahalu, S. Safran, A. Bershadsky, L. Addadi, B. Geiger, Nat. Cell Biol. 2001, 3, 466.

[7] W. H. Roos, A. Roth, J. Konle, H. Presting, E. Sackmann, J. P. Spatz, ChemPhysChem 2003, 4, 872 .

[8] N. J. Sniadecki, R. A. Desai, S. A. Ruiz, C. S. Chen, Ann. Biomed. Eng. 2006, 34, 59.

[9] M. Gupta, L. Kocgozlu, B. R. Sarangi, F. Margadant, M. Ashraf, B. Ladoux, in, Methods in cell biology,125, Ed.Paluch KE, Academic Press, 2015, p. 289.

[10] J. K. Hellmann, S. Munter, M. Kudryashev, S. Schulz, K. Heiss, A. K. Muller, K. Matuschewski, J. P. Spatz, U. S. Schwarz, F. Frischknecht, PLoS Pathog. 2011, 7, e1002080.

[11] D. Arcizet, S. Capito, M. Gorelashvili, C. Leonhardt, M. Vollmer, S. Youssef, S. Rappl, D. Heinrich, Soft Matter 2012, 8, 1473.

[12] J. L. Bargul, J. Jung, F. A. McOdimba, C. O. Omogo, V. O. Adung'a, T. Kruger, D. K. Masiga, M. Engstler, PLoS Pathog. 2016, 12, e1005448.

[13] N. Heddergott, T. Kruger, S. B. Babu, A. Wei, E. Stellamanns, S. Uppaluri, T. Pfohl, H. Stark, M. Engstler, PLoS Pathog. 2012, 8, e1003023.

[14] A. Hochstetter, T. Pfohl, Trends Parasitol. 2016, 32, 531.

[15] I. Schoen, W. Hu, E. Klotzsch, V. Vogel, Nano Lett. 2010, 10, 1823.

[16] R. Menard, J. Tavares, I. Cockburn, M. Markus, F. Zavala, R. Amino, Nat. Rev. Microbiol. 2013, 11, 701 .

[17] R. G. Douglas, R. Amino, P. Sinnis, F. Frischknecht, Trends Parasitol. 2015, 31, 357.

[18] M. Prudencio, A. Rodriguez, M. M. Mota, Nat. Rev. Microbiol. 2006, 4, 849. 
[19] M. Kudryashev, S. Munter, L. Lemgruber, G. Montagna, H. Stahlberg, K. Matuschewski, M. Meissner, M. Cyrklaff, F. Frischknecht, Cell. Microbiol. 2012, 14, 1757.

[20] A. Battista, F. Frischknecht, U. S. Schwarz, Phys. Rev. 2014, 90, 042720.

[21] C. S. Hopp, K. Chiou, D. R. Ragheb, A. Salman, S. M. Khan, A. J. Liu, P. Sinnis, eLife 2015, 4, e07789.

[22] S. Munter, B. Sabass, C. Selhuber-Unkel, M. Kudryashev, S. Hegge, U. Engel, J. P. Spatz, K. Matuschewski, U. S. Schwarz, F. Frischknecht, Cell Host Microbe 2009, 6, 551.

[23] N. Perschmann, J. K. Hellmann, F. Frischknecht, J. P. Spatz, Nano Lett. 2011, 11, 4468.

[24] J. K. Hellmann, N. Perschmann, J. P. Spatz, F. Frischknecht, Adv. Healthcare Mater. 2013, 2, 1162.

[25] I. M. Braverman, Microcirculation 1997, 4, 329.

[26] R. Amino, S. Thiberge, B. Martin, S. Celli, S. Shorte, F. Frischknecht, R. Menard, Nat. Med. 2006, 12, 220.

[27] K. S. Bane, S. Lepper, J. Kehrer, J. M. Sattler, M. Singer, M. Reinig, D. Klug, K. Heiss, J. Baum, A. K. Mueller, F. Frischknecht, PLoS Pathog. 2016, 12, e1005710.

[28] F. Frischknecht, J. Baum, Semin. Cell. Dev. Biol. 2015, 46, 78.

[29] E. J. Lupton, A. Roth, R. Patrapuvich, S. P. Maher, N. Singh, J. Sattabongkot, J. H. Adams, Parasitol. Int. 2015, 64, 211.

[30] R. Amino, B. Franke-Fayard, C. Janse, A. Waters, R. Menard, F. Frischknecht, in, Imaging cellular and molecular biological functions, Eds:Spencer LS, Frischknecht F, Springer Berlin Verlag, 2007, p. 345.

[31] M. Kudryashev, S. Lepper, R. Stanway, S. Bohn, W. Baumeister, M. Cyrklaff, F. Frischknecht, Cell. Microbiol. 2010, 12, 362.

[32] S. Hegge, S. Munter, M. Steinbuchel, K. Heiss, U. Engel, K. Matuschewski, F. Frischknecht, FASEB J. 2010, 24, 2222.

[33] J. P. Vanderberg, J. Protozool. 1974, 21, 527.

[34] F. Frischknecht, P. Baldacci, B. Martin, C. Zimmer, S. Thiberge, J. C. Olivo-Marin, S. L. Shorte, R. Menard, Cell. Microbiol. 2004, 6, 687.

[35] M. Cyrklaff, M. Kudryashev, A. Leis, K. Leonard, W. Baumeister, R. Menard, M. Meissner, F. Frischknecht, J. Exp. Med. 2007, 204, 1281.

[36] G. N. Montagna, C. A. Buscaglia, S. Münter, C. Goosmann, F. Frischknecht, V. Brinkmann, K. Matuschewski, J. Biol. Chem. 2012, 287, 2410.

[37] A. F. Carey, M. Singer, D. Bargieri, S. Thiberge, F. Frischknecht, R. Menard, R. Amino, Cell. Microbiol. 2014, 16, 768. 
[38] C. W. Kuo, D. Y. Chueh, P. Chen, J. Nanobiotechnol. 2014, 12, 54.

[39] T. Maier, T. Haraszti, PLoS One 2015, 10, e0136432.

[40] R. Natarajan, V. Thathy, M. M. Mota, J. C. Hafalla, R. Menard, K. D. Vernick, Cell. Microbiol. 2001, 3, 371.

[41] S. Hegge, M. Kudryashev, A. Smith, F. Frischknecht, Biotechnol J 2009, 4, 903. 

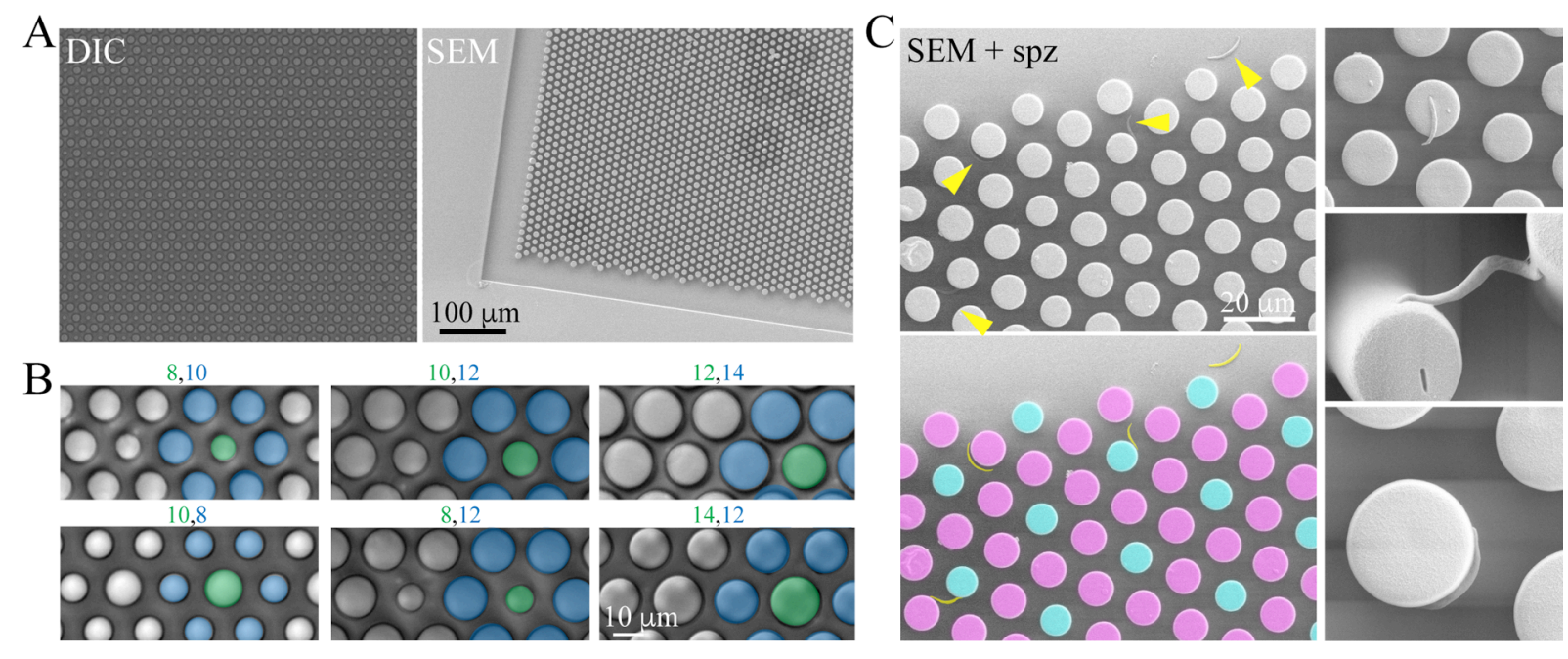

Figure 1

Pillar arrays with mixed diameter pillars.

(A) Mixed pillar arrays as seen by differential interference contrast (DIC) or scanning electron microscopy (SEM). Scale bar: $100 \mu \mathrm{m}$.

(B) Examples of mixed pillar arrays as seen from the top by DIC. The central pillar is marked in green and the surrounding pillars are marked in blue. The corresponding pillar diameter is indicated in the matching colour. The pillar arrays at the left $(8,10$ and 10,8$)$ and right $(12,14$ and 14,12) are termed coupled arrays. Scale bar: $10 \mu \mathrm{m}$.

(C) Scanning electron micrographs of the edge of a mixed-pillar array (left) with four sporozoites (yellow arrowheads) indicated. Sporozoites are coloured in yellow in the false colour image at the bottom. The pillars of the different sizes are coloured cyan (thin pillars) and magenta (thick pillars). Scale bar: $20 \mu \mathrm{m}$. SEM images of sporozoite bound to the top or side of pillars are shown on the right at various magnifications. 


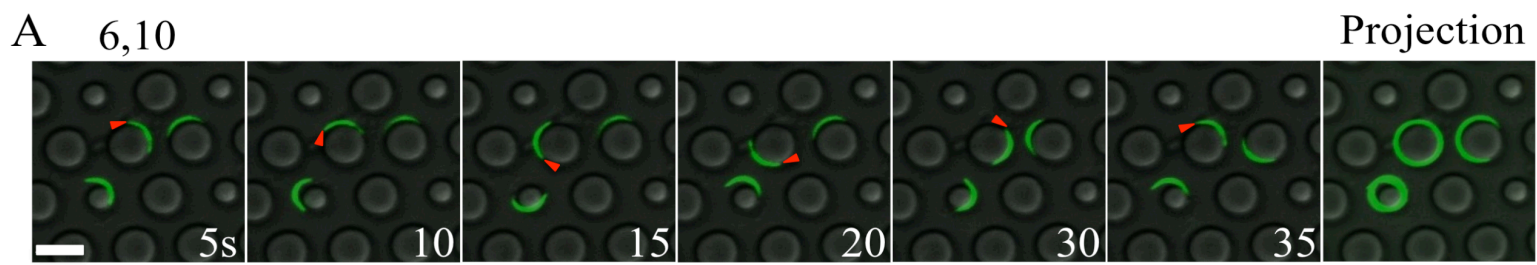

10,14
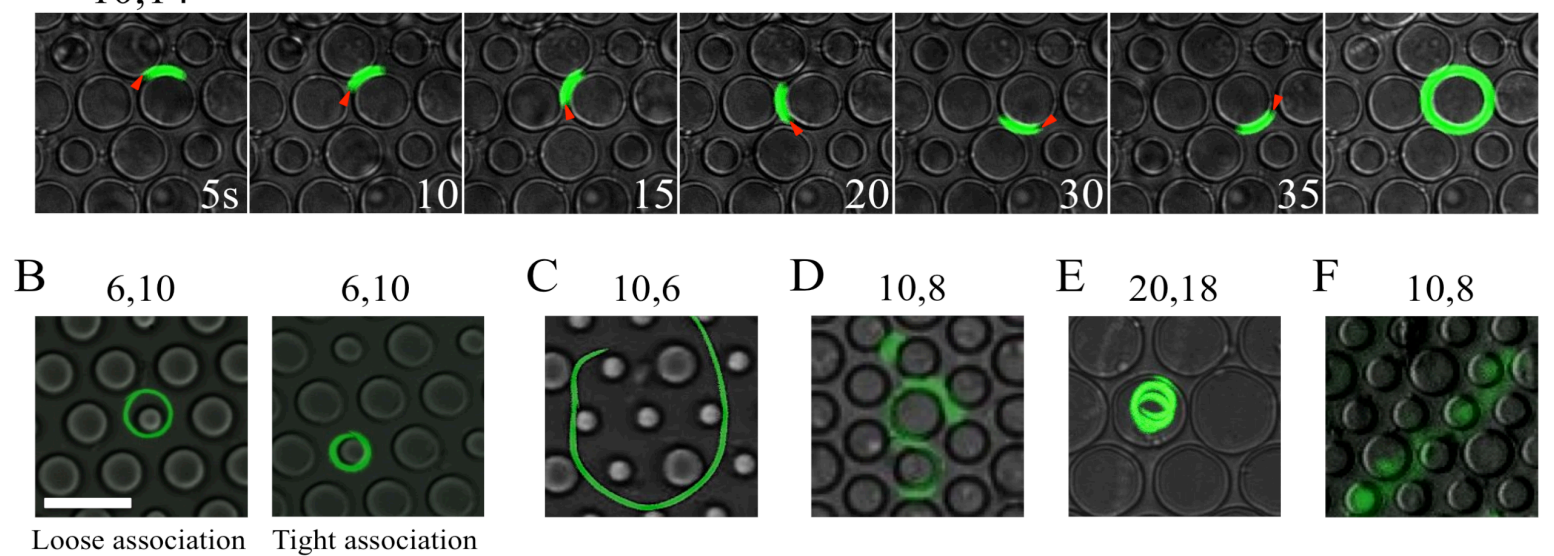

Figure 2

Examples of parasite behavior in mixed pillar arrays.

(A) P. berghei sporozoites expressing cytoplasmic GFP migrating around pillars in different mixed pillar arrays of pillars with a diameter of 6 and $10 \mu \mathrm{m}$ as well as 10 and $14 \mu \mathrm{m}$, respectively. White numbers indicate time in seconds and black numbers indicate the pillar diameter in the respective arrays. Maximum fluorescent projections over several images highlighting the path of the motile parasites are shown at the right. Scale bar: $10 \mu \mathrm{m}$.

(B) Maximum fluorescent intensity projections of sporozoites moving around $6 \mu \mathrm{m}$ pillars either without a contact to the pillar (left panel) or in close association with the pillar (right panel). Scale bar: $20 \mu \mathrm{m}$.

(C) Projection of a sporozoite moving around seven pillars of 10 and $6 \mu \mathrm{m}$ diameter.

(D) Projection of a sporozoite switching from a $10 \mu \mathrm{m}$ to an $8 \mu \mathrm{m}$ pillar.

(E) Projection of a sporozoite moving on top of a large pillar with $18 \mu \mathrm{m}$ diameter. 
(F) Projection of a sporozoite floating on top of the pillar array. Note that a sporozoite positioned on top of a pillar leads to a fluorescent flare around the pillar circumference (see Movie S3). This can be seen in all tested arrays. 
A

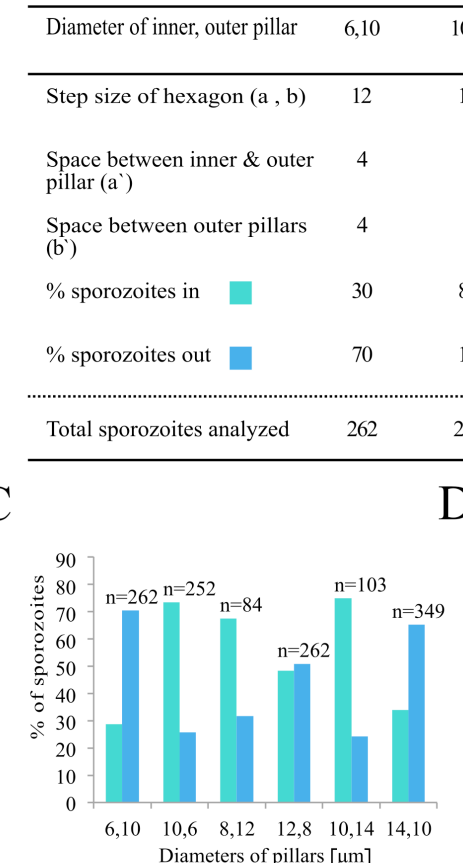

$\begin{array}{lllllllll}10,6 & 10,8 & 8,12 & 12,8 & 12,10 & 12,14 & 14,12 & 10,14 & 14,10\end{array}$

$\begin{array}{llllllll}14 ; 15 & 17 & 13 & 15 & 18 ; 16 & 15 ; 16 & 16 ; 15 & 16 ; 15\end{array}$

$\begin{array}{lllllllll}4 & 5 ; 6 & 3 & 3 & 4 & 3 & 3 & 4 ; 3 & 4 ; 3\end{array}$

$\begin{array}{lllllllll}4 & 5 ; 4 & 5 & 5 & 5 & 4 & 4 & 2 ; 5 & 6 ; 5\end{array}$

$\begin{array}{lllllllll}82 & 49 & 68 & 49 & 40 & 56 & 34 & 83 & 34\end{array}$

$\begin{array}{lllllllll}18 & 51 & 32 & 51 & 60 & 44 & 66 & 17 & 66\end{array}$

$\begin{array}{lllllllll}252 & 658 & 84 & 262 & 91 & 52 & 430 & 56 & 349\end{array}$

$\mathrm{D}$

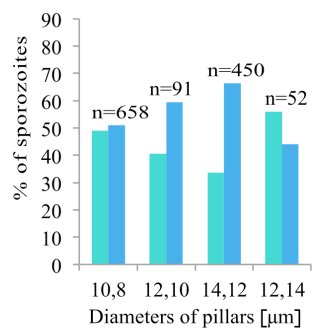

Sporozoites moving around inner pillars outer pillars
B

(i)

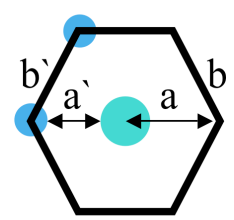

(ii)

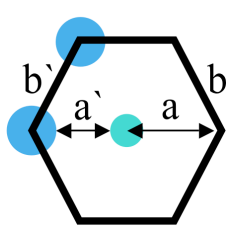

$\mathrm{F}$
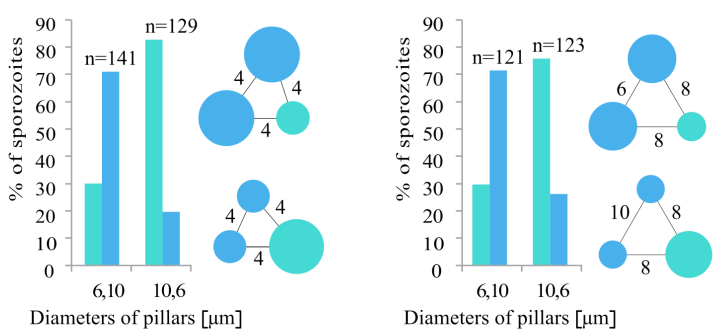

Figure 3

Sporozoite association to pillars of different diameters

(A) Table showing the data of differently fabricated pillar arrays along with schematics illustrating the pillar arrangement.

(B) Graphical depiction of pillar array unit. The step sizes of the pillar-to-pillar distances as measured from either the pillar centers $(\mathrm{a} ; \mathrm{b})$ or the pillar circumferences $\left(\mathrm{a}^{\prime} ; \mathrm{b}^{\prime}\right)$ are indicated. Note that $\mathrm{a}=\mathrm{b}$. Blue represents the outer pillars and turquoise represents the central pillars also for panels C-F.

(C) Graphical representation of $P$. berghei sporozoite movement around pillars in three different coupled arrays, where the thicker pillar of the same (i.e. 10, 12 and $14 \mu \mathrm{m}$ ) diameter was either surrounded by the thinner pillar (i.e. 6,8 and $10 \mu \mathrm{m}$, respectively) or vice versa. Note that most sporozoites moving around $6 \mu \mathrm{m}$ pillars are not tightly associated with the pillar. Here all sporozoites moving tightly around $6 \mu \mathrm{m}$ pillars are counted. The number of parasites is adjusted for the three-fold bias of the surrounding pillars over the central ones. 
Only sporozoites circling around pillars were enumerated. All differences of pairs other then the '12,8' pair are highly significant ( $\mathrm{p}<0.0001)$; Fischers exact test.

(D) Quantitative analysis of P. berghei sporozoite movement around pillars from different arrays with pillar diameter differences of just $2 \mu \mathrm{m}$. The number of parasites is adjusted for the three-fold bias of the surrounding pillars over the central ones. Note the bias towards the $12 \mu \mathrm{m}$ pillars in the $12 / 14$ arrays. All differences of pairs other than the '10,8' pair are highly significant $(\mathrm{p}<0.01)$; Fischers exact test.

(E, F) Quantitative analysis of the proportion of sporozoites moving around pillars in 10 and 6 $\mu \mathrm{m}$ reciprocal arrays with a uniform pillar-to-pillar distance of $4 \mu \mathrm{m}(\mathrm{E})$ and in arrays with varied pillar-to-pillar distances of 10 or $8 \mu \mathrm{m}(\mathrm{F})$. Data from panel $\mathrm{E}$ are a subset of that represented in panel $\mathrm{C}$ and performed at the same time as those in panel F. All differences are highly significant $(\mathrm{p}<0.001)$; Fischers exact test. 

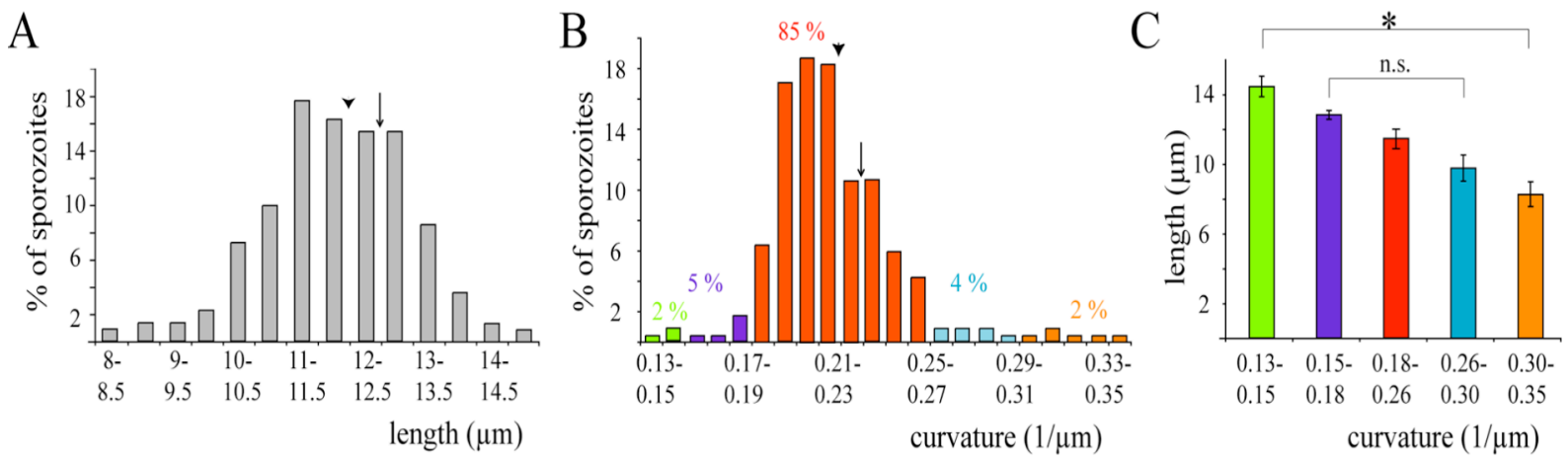

Figure 4

Long sporozoites show reduced curvature

(A, B) Distribution of $P$. berghei sporozoite lengths (A) and curvatures (B) from automated analysis $(n=973)$. The arrowheads indicate the arithmetic mean. Colours in B represent populations analyzed in $\mathrm{C}$ and indicate the respective percentage from the entire population. The arrows in A and B indicate the mean of P. yoelii sporozoites.

(C) Inverse correlation of sporozoite lengths and curvatures at the extreme ends. Colours of the different curvature ranges are the same as those plotted in B. Hence the green bar corresponds to only $2 \%$ of the population, while the red bar corresponds to $85 \%$ of the population; see also Figure S2. 
A

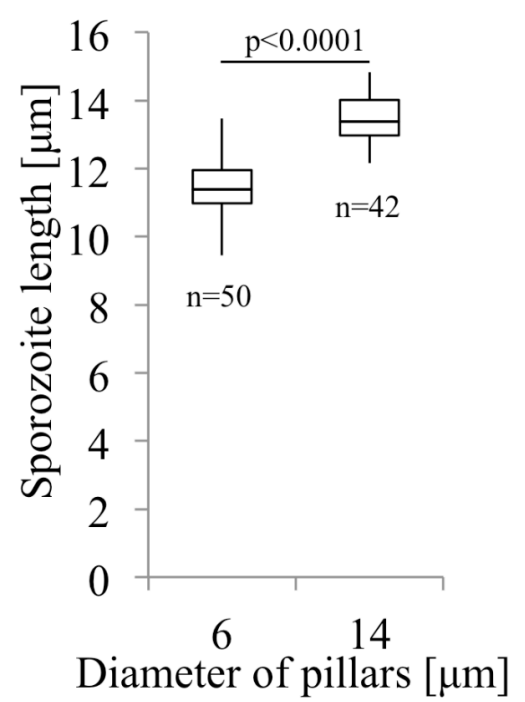

B

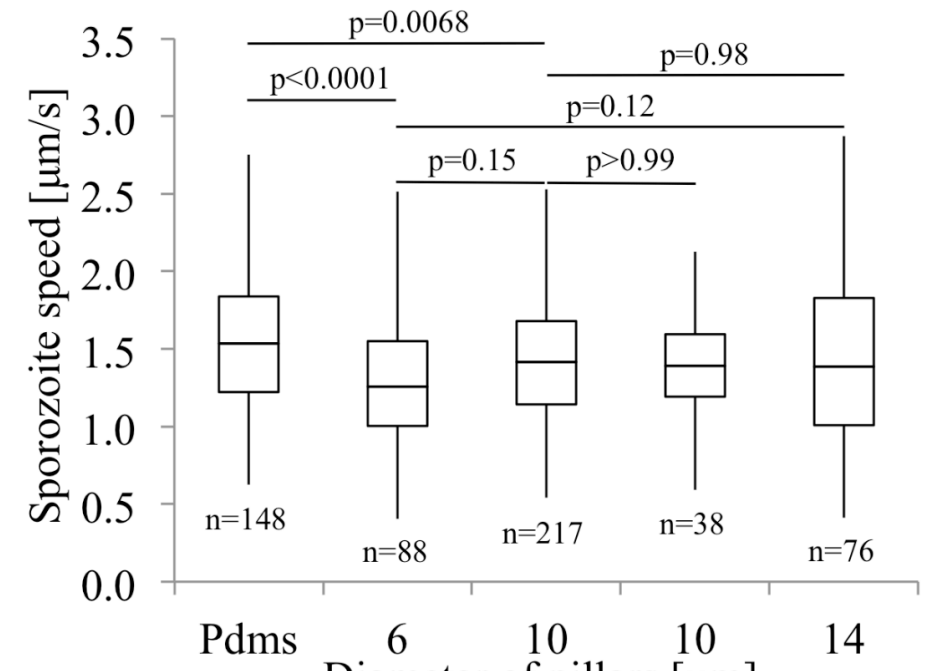

Figure 5

Sporozoite length, speed and curvature

(A) Length of sporozoites circling tightly around 6 and $14 \mu \mathrm{m}$ pillars. The differences are highly significant $(\mathrm{p}<0.001)$ using a student's t-test.

(B) Speed distribution of $P$. berghei sporozoites moving on PDMS or circling around pillar pairs of 6 and $10 \mu \mathrm{m}$ diameter or 10 and $14 \mu \mathrm{m}$ diameter, respectively. The differences between PDMS, 6 and $10 \mu \mathrm{m}$ pillars is significant $(\mathrm{p}<0.05)$; one-way Anova. 
A

\begin{tabular}{lcc}
\hline $\begin{array}{lcc}\text { Pillar } \\
\text { diameter }\end{array}$ & $\begin{array}{c}18, \\
20\end{array}$ & $\begin{array}{c}20, \\
\end{array}$ \\
\hline a & 22 & 22 \\
a & 3 & 3 \\
& & \\
b' & 2 & 4
\end{tabular}

B

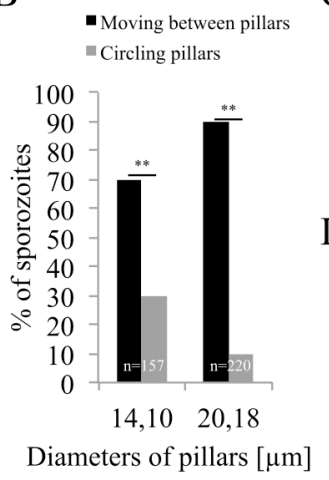

$\mathrm{C}$

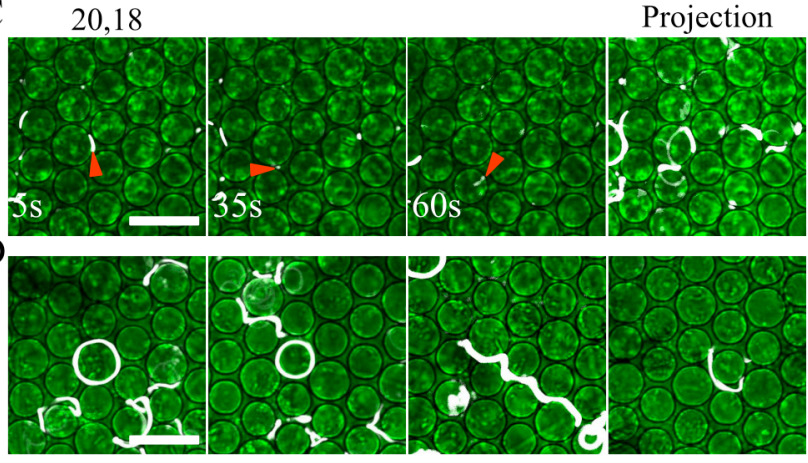

Circling sporozoites

Linear and diving sporozoites

Figure 6

Sporozoite association with very thick pillars

(A) Table showing the step size of the designed hexagonal arrays of very thick pillars. The pillar-to-pillar distance as measured from the pillar center is labelled as $(a ; b)$, the distance between pillar circumferences labelled as (a'; b') as in Figure 3.

(B) Comparative analysis of the selection behavior of sporozoites in 14,10 and $20,18 \mu \mathrm{m}$ arrays. Note that fewer parasites move around pillars with larger diameters. The difference between sporozoites moving around and between is significant $(p<0.001)$; Fischers exact test. (C) Sporozoites migrating within 18 and $20 \mu \mathrm{m}$ pillars. White numbers indicate time in seconds and black numbers indicate the pillar diameter in the arrays. The projection images are maximum fluorescent projections over several images highlighting the path of the motile parasites. Scale bar: $20 \mu \mathrm{m}$; red arrows point at the apical tip of the sporozoite.

(D) Projections of sporozoites migrating around the different pillars, linearly gliding and 'diving' into the arrays; red arrows point at the apical tip of the sporozoite. Scale bar: $20 \mu \mathrm{m}$. 

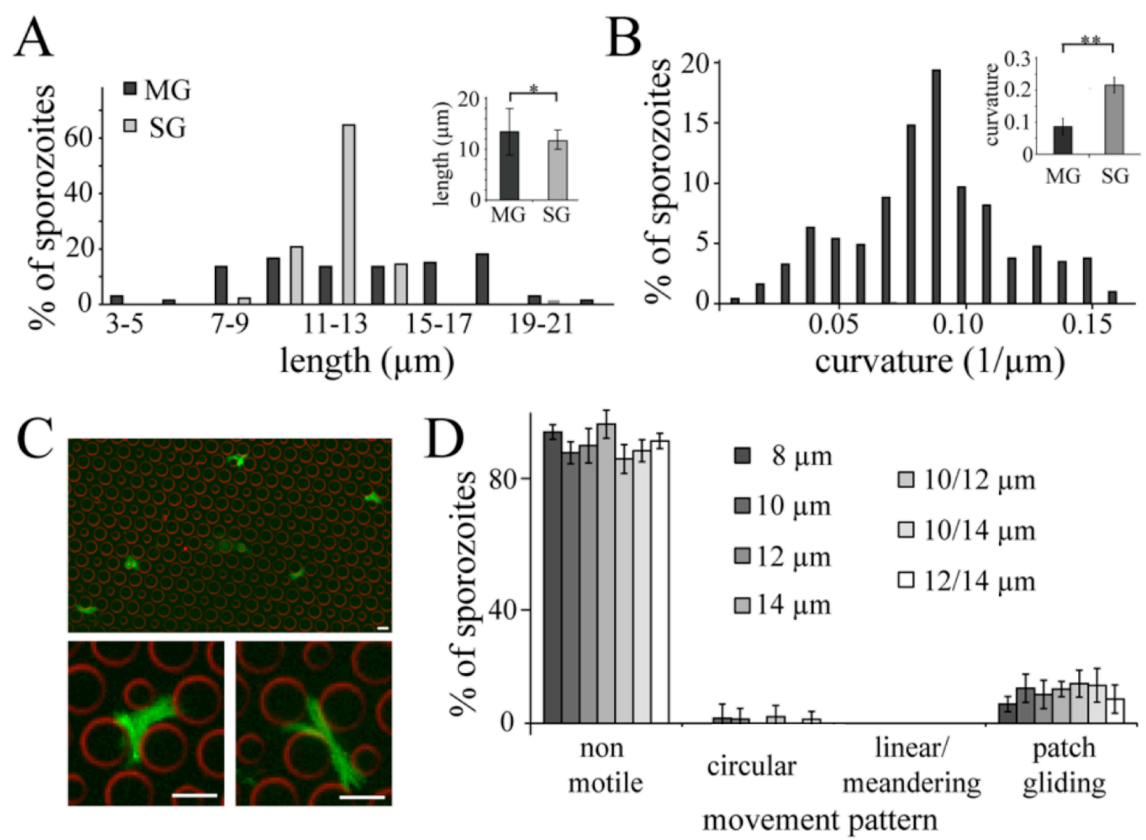

$\mathrm{E}$

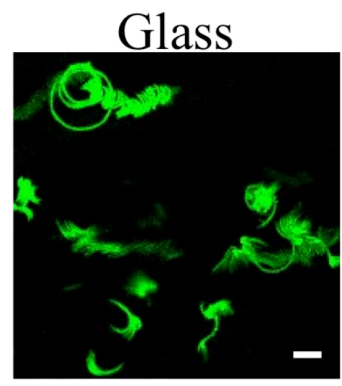

Coronin (-)

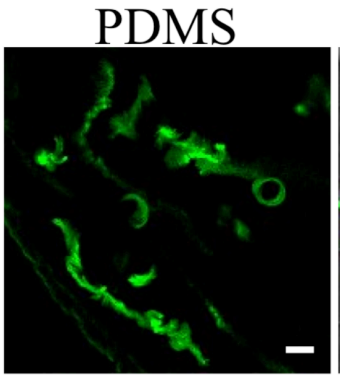

Coronin (-)
Pillars

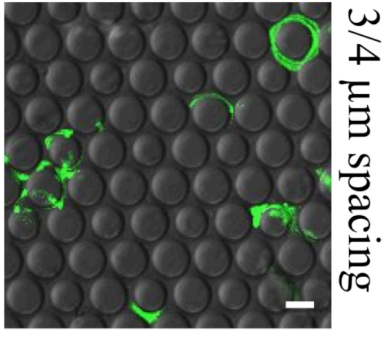

Coronin (-)

Figure 7

Non-motile sporozoites are migrating in pillar arrays

$(\mathrm{A}, \mathrm{B})$ Distribution of midgut (dark bars, $\mathrm{MG} ; \mathrm{n}=187$ ) and salivary gland (light bars, $\mathrm{SG} ; \mathrm{n}=$ 973) derived sporozoite length and curvature. Note that salivary gland derived sporozoites are more curved, while midgut derived sporozoites are slightly longer.

(C) Fluorescent intensity projections of midgut derived sporozoites. Note the difference of their movement pattern compared to salivary gland derived sporozoites that were used throughout the study. The patterns at the bottom reflect patch-gliding sporozoites. Scale bars: $10 \mu \mathrm{m}$. 
(D) Quantitative assessment of sporozoite movement pattern from midgut-derived sporozoites $(n=264)$. Note that most are not motile across a range of pillar arrays.

(E) Maximum fluorescence intensity projections of coronin(-) sporozoites placed on glass, PDMS and within a pillar array of 12 and $14 \mu \mathrm{m}$ pillar diameter and 3 and $4 \mu \mathrm{m}$ pillar-topillar distance. Of 545 analyzed sporozoites, 11\% of sporozoites moved robustly on glass, while on PDMS 14\% did and in the paired pillar array $8 \%$. There was no significant difference between these numbers; $(\mathrm{p}=0.1)$; Chi-square $\left(\mathrm{X}^{2}, 4.583 ; \mathrm{df}, 2\right)$ test. 


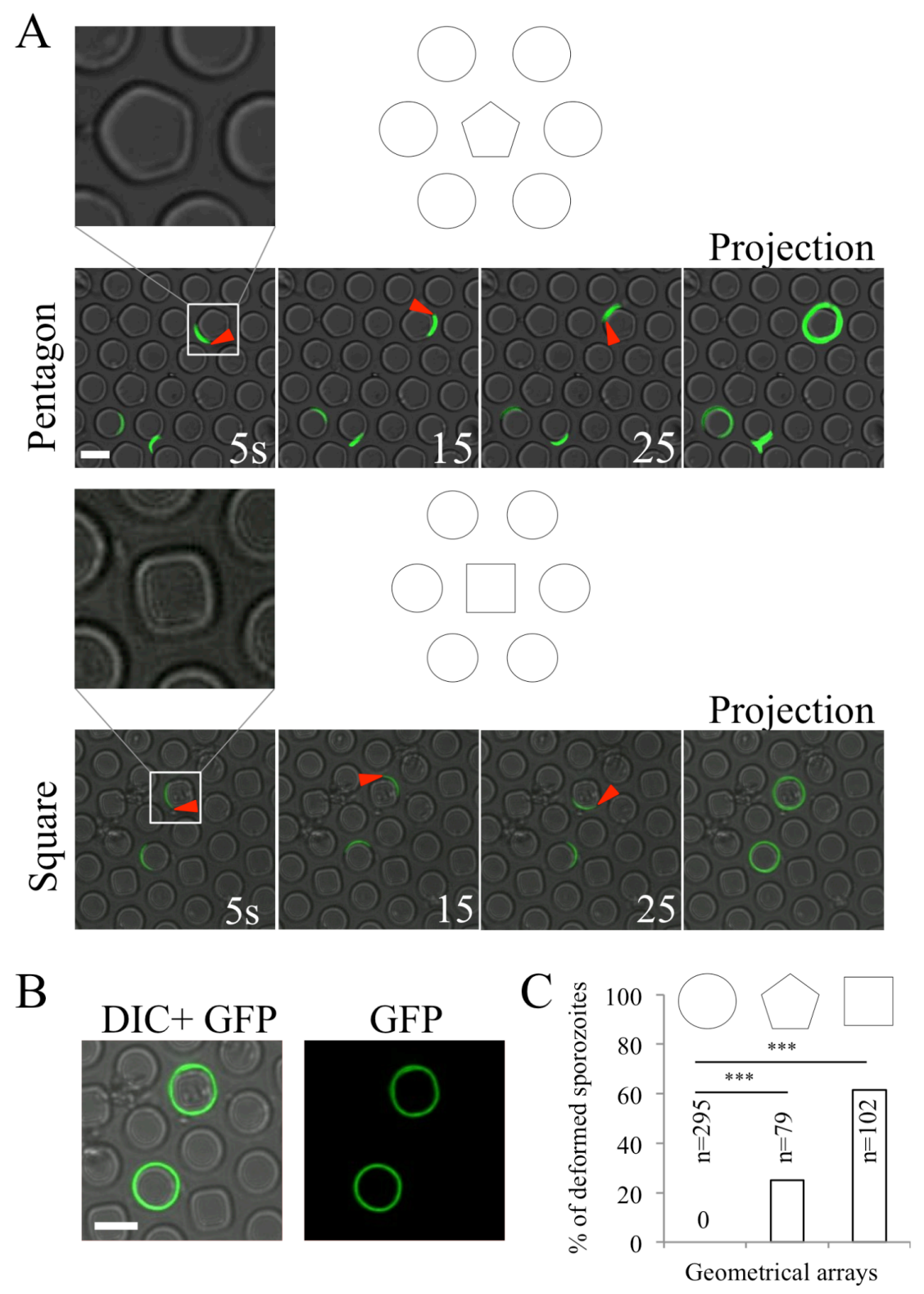

Figure 8

Sporozoite motility in geometrical arrays

(A) A DIC image and cartoon of the arrays featuring pentagon and square-shaped pillars as well as time series of sporozoites migrating around pentagonal and square pillars. The circular pillar is $10 \mu \mathrm{m}$ in diameter for both arrays, while the pentagons and squares are $11 \mu \mathrm{m}$ wide. White numbers indicate time in seconds. The projections images are maximum fluorescent projections over several images highlighting the path of the motile parasites; red arrows point at the apical tip of the sporozoite. Scale bar: $10 \mu \mathrm{m}$. 
(B) Fluorescent intensity projection of two sporozoites with one showing a deformed trajectory around a squared pillar. Scale bar: $10 \mu \mathrm{m}$.

(C) Proportions of sporozoites changing their shape from their usual curvature while moving around pillars shaped as circles, pentagons or squares. Parasites were scored as deformed if they changed their shape at least once during 50 seconds. Total number of sporozoites moving around the respective pillars are indicated. The difference is highly significant $(p<0.0001)$; Chi-square $\left(X^{2}, 204.9 ;\right.$ df, 2) test. 